\title{
IDENTIFICATION OF TECHNOLOGY-RELEVANT ENTITIES BASED ON TREND CURVES AND SEMANTIC SIMILARITIES
}

\author{
Sukhwan Jung, Rachana Reddy Kandadi, Rituparna Datta, \\ Ryan Benton and Aviv Segev
}

Department of Computer Science, University of South Alabama, Mobile, Alabama, USA

\begin{abstract}
Technological developments are not isolated and are influenced not only by similar technologies but also by many entities, which are sometimes unforeseen by the experts in the field. The authors propose a method for identifying technology-relevant entities with trend curve analysis. The method first utilizes the tangential connection between terms in the encyclopedic dataset to extract technology-related entities with varying relation distances. Changes in their term frequencies within 389 million academic articles and 60 billion web pages are then analyzed to identify technology-relevant entities, incorporating the degrees and changes in both academic interests and public recognitions. The analysis is performed to find entities both significant and relevant to the technology of interest, resulting in the discovery of 40 and 39 technologyrelevant entities, respectively, for unmanned aerial vehicle and hyperspectral imaging with 0.875 and 0.5385 accuracies. The case study showed the proposed method can capture hidden relationships between seemingly unrelated entities.
\end{abstract}

\section{KEYWORDS}

Technology Forecasting, Trend Curve, Big Data, Academic Articles, Web Pages

\section{INTRODUCTION}

Identification of relevant terms for a specific technology plays a crucial role in technology funds and government research grants, allowing them to better direct their investments to encourage technological developments beneficial to the target technology. It is also one of the main research fields for stock market prediction as technology development directions affect the stock markets. The current work proposes an approach for identifying any type of entities relevant to the given technology, based on the trend curves of related entities found from recursive encyclopedic connections to the technology. This perspective offers a novel approach of technology trend analysis, granting a possibility of detecting seemingly unrelated entities that cannot be found with conventional means.

The proposed method offers a means of identifying significant entities relevant to a given technology based on term frequency and degree of usage growth. It analyzes technology- relevant entities from Wikipedia in the whole domain of academic articles (academia) and web pages (web) with the help of Google search engine, incorporating both the academic interests and public recognitions of the given entities, each representing the earliest and the latest predictive time windows. Wikipedia, an online encyclopedia with built-in page links between related articles, allows the extraction of not only the related technologies but also any related entities, providing generalizability to the proposed method if desired. The use of Wikipedia also allows the use of document similarities when filtering for entities with more relevance to the target technology. 
International Journal of Web \& Semantic Technology (IJWesT) Vol.11, No.1/2/3, July 2020

The authors previously showed technology trends in different datasets contain distinct patterns while sharing an overall shape on different time windows [1]. The comparison of the proposed method on two datasets analyzes the differences in the list of entities deemed relevant to them. In addition, the analysis of entities common in both datasets and their respective trend curves presents an integrated view of the technology-relevant entities over multiple dimensions.

The main contributions of this work are as follows:

- On an algorithmic level, the authors provide an implementation of the proposed method based on the academia and web.

- On a conceptual level, the authors propose a multi-domain approach for identifying any entities relevant to a specific technology based on term frequency and moving gradient, which can be semantically and syntactically distant from the technology. Preliminary experimentation validated that the performance can be enhanced with the introduction of natural language processing as well.

- On a practical level, the authors identify a list of relevant, possibly hidden, entities for the target technology and how different datasets contribute to the result.

Section 2 reviews the related work on technology forecasting with regard to the necessity of normative approaches based on technological curves and their limitations. Section 3 explains the proposed method and experiment in detail. The experiment results in Section 4 show that the proposed method can identify entities related to the given technology with hidden relationships, and Section 5 states the concluding remarks and future work.

\section{RELATED WORK}

The traditional approach for technology forecasting is a manual approach, including scenario building [2], forecast by analogy [3], and the Delphi method [4]. Scenario building lets analysts generate a series of plausible scenarios with both optimistic and pessimistic developments; these developments aim to be compatible, with substantial effects, with unlikely events that are often disregarded in other methods. Forecast by analogy employs analogical comparison between the known phenomena and the technology trends with the assumption they behave similarly. The Delphi method is a more structured technique, first developed as a systematic and interactive method of forecasting. It relies on a consensus among a panel of experts, which is reached by repeated rounds of questionnaires to the participating experts. The belief is that the variance of the answers will decrease with each iteration and the group will converge towards an answer that can be regarded as correct. The process ends once it either reaches a certain number of rounds or achieves a steady consensus; the answers from the final round determine the result. These manual methods often require a large amount of contribution from numerous field-related experts and hence are expensive to utilize, but still have been used in recent years [5] for its high domain adaptability.

Normative methods such as morphological models [6] and mission flow diagrams [7] are complementary to such processes that attempt to automatically project future behaviors from past data. Based on systems analysis, normative methods view future needs in the field as the scheduled progress of the field and predict future behaviors based on them [7]. Extrapolations on past data are used to analyze changes in the popularity or intensity of a given topic, which can be matched into estimation lines such as linear, polynomial, exponential, and parabolic lines [8]. Extrapolation on a pre-defined technological curve is widely used as well, matching the past data to estimations lines such as Gartner's hype cycle or other technological growth 
International Journal of Web \& Semantic Technology (IJWesT) Vol.11, No.1/2/3, July 2020

curves such as S-curve [9]. The future technological stages are then predicted upon the estimation line. The limitations of normative methods suggested in more recent years include incongruencies found from the Gartner dataset and its hype cycle [10] and less generalizability for different technology fields. This indicates that both the manual and automatic methods lack the ability to be implemented in related technological fields [11].

Other fields of research tried to address related fields to generate better technology forecasts. The content transition from one topic to another during topic evolution is identified in the form of complementary trend curve patterns, connecting multiple technologies in transitional states [12], [13]. The topics are extracted statistically from a document collection, and the popularity trend curve of each topic is generated by connecting their popularities in discretely divided document subsets per timeslots. The content transition between topics is identified when one topic experiences a significant drop in popularity when the other topic experiences a significant increase, which is translated as the former topic being transferred to the latter one. Technology diffusion can be used for a more specific case of technology transfer where the one is replaced by another, such as LED is replaced by OLED for the TV screen market [14]. The inconsistency problem remains, however; the technology trend curves can vary for different forecast methods and datasets on which they are used. Combining different forecasts of the same technology allows remediation of the disadvantages from individual forecasts at the potential expense of individual advantages [8]. The authors' previous work utilized a combination of forecasts from various datasets to show that the predictive power of different forecasts varies based on the nature of the dataset used. Changes in technology term frequencies in public datasets such as news, books, and web pages are preceded by more academic datasets such as academic articles and patents, resulting in a longer predictive time window for technology growth prediction [1].

The traditional manual approach for technology forecasting requires an extensive amount of time and resources, and cheaper alternatives are highly sought after. Normative methods extrapolate on predefined technological growth curves were successful in forecasting technological development within a given technology field. They showed limited performance in forecasting related technologies. Different technologies do not necessarily follow the same growth curve. However, our work proposes a more generic forecasting model for automatic technology forecasting.

\section{METHOD}

The proposed method is based on analyzing the frequency trends of technology-relevant entities on academia and web each representing two different dataset orientations - academic and public. Documents in both datasets are timestamped by their publication date and can be sequentially discretized. The method consists of 1) extraction of technology-related entities having recursive encyclopedic connections to the technology in question, and 2) identification of technologyrelevant entities through the entity filtering with their timeline trend curves over two different datasets, incorporating both academic and public interests. The analysis was performed for two selected technologies, Unmanned Aerial Vehicle (UAV) and Hyperspectral Imaging (HSI), identifying relevant entities from Wikipedia articles using the entirety of academia and web. In addition, the entities were evaluated manually to showcase the necessity of multi-datasets and the possible applications of the proposed method.

\subsection{Extracting Technology-Related Entities}

The technology-related entities were extracted based on the Wikipedia articles. The semistructured nature of the articles allow multiple extraction approaches; advanced natural language processing such as context recognition can be used to extract terms from the unstructured texts 
from the articles [15], structured data such as infobox tables or links can be utilized to extract pre-defined terms, and the articles can be read to manually identify the related entities. The seealso section of the Wikipedia article is a list of internal pagelinks manually written by participants and moderators. The see-also section was used in this experiment as its semi-structured nature allows the extracted terms to be not limited to specific contents, domains, or types while providing human-verified semantic, syntactic, or conceptual connections between the original and linked articles.

Given a specified technology, such as UAV, its related entities are extracted by recursively parsing the Wikipedia articles starting from its article. The dedicated library for reading Wikipedia ${ }^{1}$ is not ideal when multiple articles are considered, and a different approach is utilized instead. The Wikipedia article is retrieved via the webserver using a specified URL which is then processed with a Python library BeautifulSoup ${ }^{2}$ to extract HTML snippet for the see-also section and the pagelinks contained within it. The articles from the collected pagelinks become the first set of technology-related entities with a distance of 1 from the seed article. The algorithm is then run recursively on the extracted articles for breadth-first entity extraction; the see-also sections for articles with distance $=n$ are extracted to get entities with distance $=n+1$. The recursive search result in exponential growth is the number of entities found.

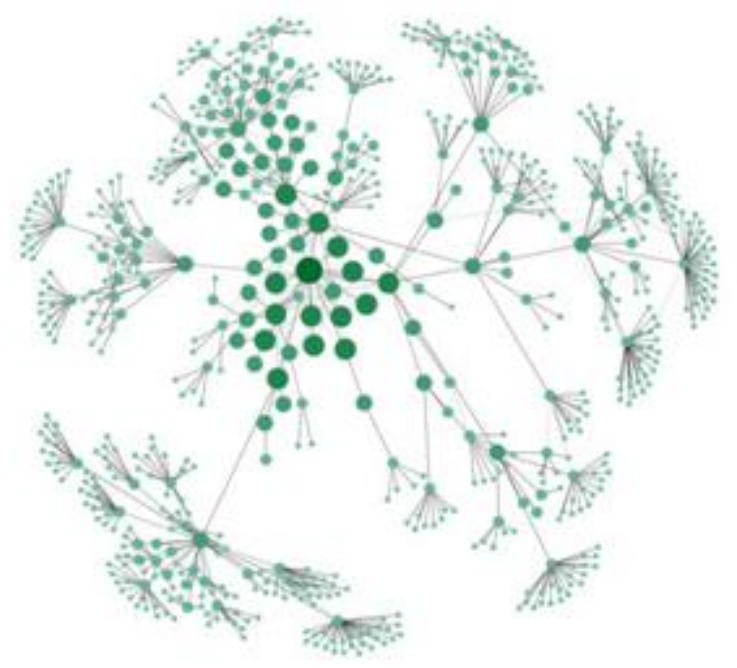

Figure 1(a). Related to $U A V$.

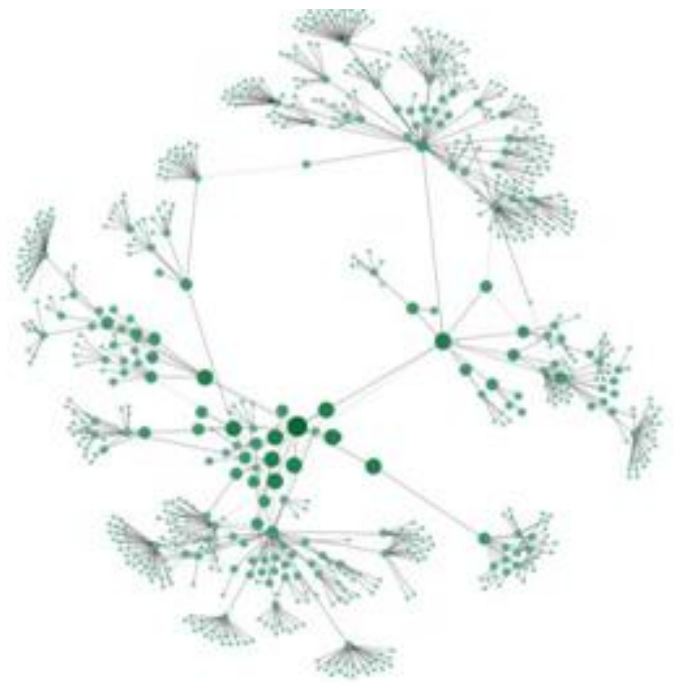

Figure 1(b). Related to HSI.

Figure 1. Visualizations of technology-related entities connected by see-also relationships with diminishing node size and color for entities more distant from the given technology.

Examples of entities with distance $\leq 4$ are displayed in Figure 1(a) and Figure 1(b) based on two technologies, with articles as nodes and see-also connection as links. The node size and color intensity reflect the distance from the root node, and the graph shows mostly tree structures with only a fraction of the links between branches; such a link indicates that the articles were inversely connected. They show the majority of entities, $71.39 \%$ for $U A V$ and $76.49 \%$ for $H S I$, are the furthest from the technology with distance $=4$. The exhaustive search can be done for longer distances for more than a quarter of million entities but is impractical; the authors used the first 500 results as the technologyrelated entities which can be satisfied with distance $\leq 4$. The pseudocode for extracting technology-related entities is shown in Figure 2, where articles are recursively searched 
until the given number of related articles, 500 in the experiment, are collected. Breadthfirst search is done for each of the links in the articles' see-also section. The possibility of cycling is removed by only accessing newly-met articles in the process.

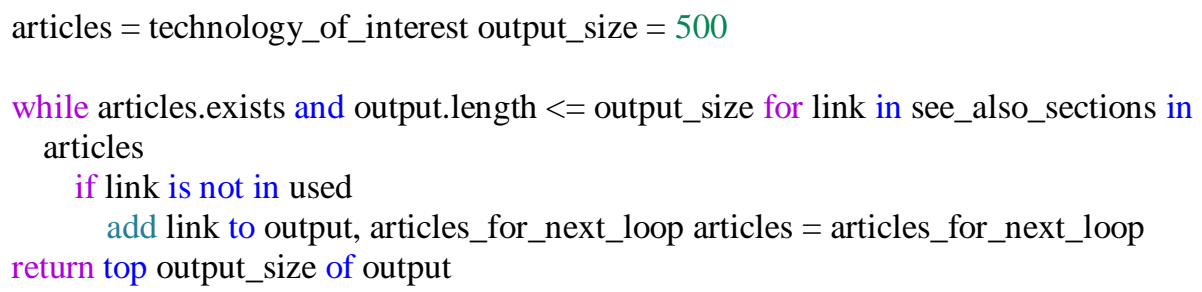

Figure 2. Pseudocode for Extracting Technology-Related Entities.

\subsection{Extracting Technology-Related Entity Trends}

The next step is the extraction of trend curves of the list of the entities found from the previous step. This is achieved by extracting their term frequencies in the massive document collections at discrete timeslots, which, in this study, was yearly intervals. The whole domains of academia and $w e b$ were chosen as the document collections in the experiments. The sheer volume of research publications of the WWW hinders effective searching, and the Google search engine is utilized which searches the documents indexed by Google, respectively exceeding 389 million articles [16] and 60 billion pages [17]. This allowed utilization of Google search engine APIs during the trend curve extraction process, where each data point is the number of search results in a given year. The search result for academia is the number of academic articles containing the term in their titles and abstracts; when the Google API can access full text it is used instead. For web, the total number of webpages containing the term is used instead. The trend curves are generated by connecting the discrete data points into a series of line graphs. The trend curves are not normalized as in the previous research [18] since the process searches not only for curves with a specific growth pattern but also curves with overall elevated values. All entities are deemed related to the technology in question and are treated equally regardless of their distances from it, or the number of see-also sections between them.

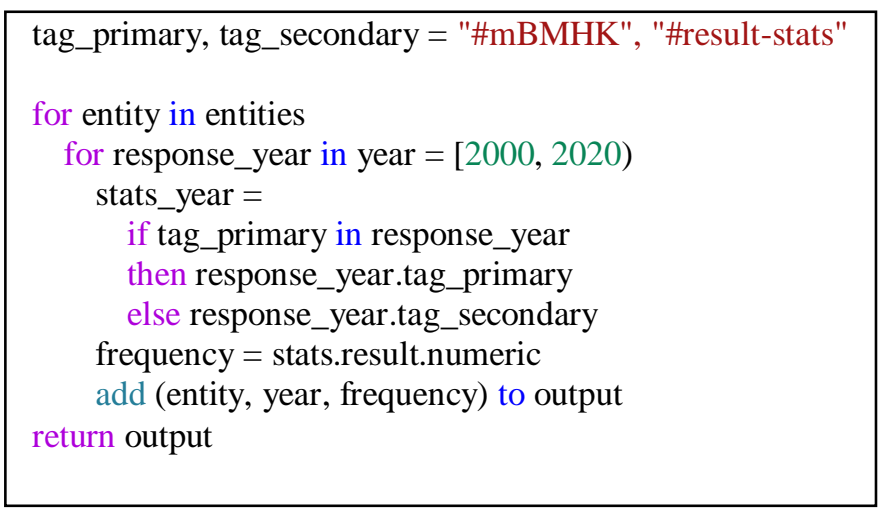

Figure 3. Pseudocode for Extracting Technology-Related Entity Trends.

Figure 3 shows the pseudocode for the entity trends extraction process. For each entity obtained in the previous section, the Google search result in HTML format is retrieved for every year from 2000 to 2019. The statistical metadata of the response is stored within an HTML div tag identifiable by two possible ids, result-stats and $m B M H K$, which is extracted as a snippet. The 
search result count within the snippet is then extracted and stored as the frequency for the year. The only difference between academia and web is the structure of the URL the Google search engine requires; therefore the same implementation is used for both. The number of calls to the Google search engine is limited to 100 per day, and queries were required to be made every 100 seconds.

Results of entity trends extraction are shown in Figure 4 with four graphs. Entities related to both the $U A V$ and HSI share similar patterns, diminishing towards the year 2019 after plateauing at around 2010 in academia in Figure 4(a) and Figure 4(c) while showing exponential growth in web in Figure 4(b) and Figure 4(d). This suggests the entities related to both technologies are experiencing initial hype with the public while the researchers have already passed this stage and show diminished interests in the same entities. Such differences are validated by the authors' previous research on the different time windows for technology growth curves in different datasets, where the technology's development starts with the academic domain and the public inherits the changes afterward [1], [18].

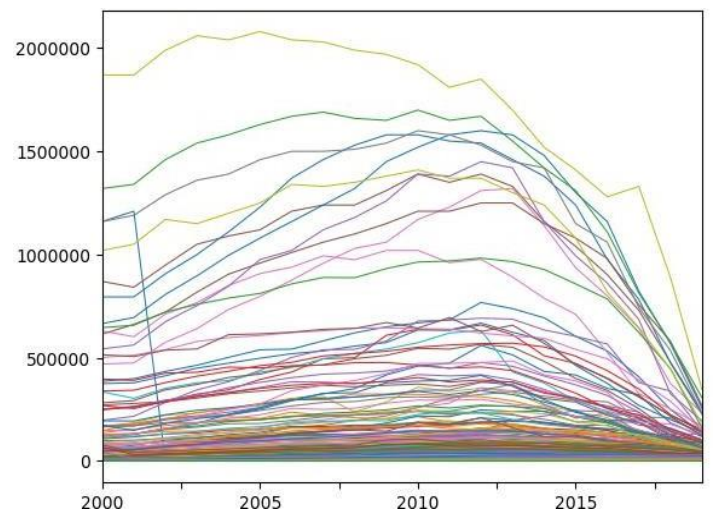

Figure 4(a). For $U A V$ in academia.

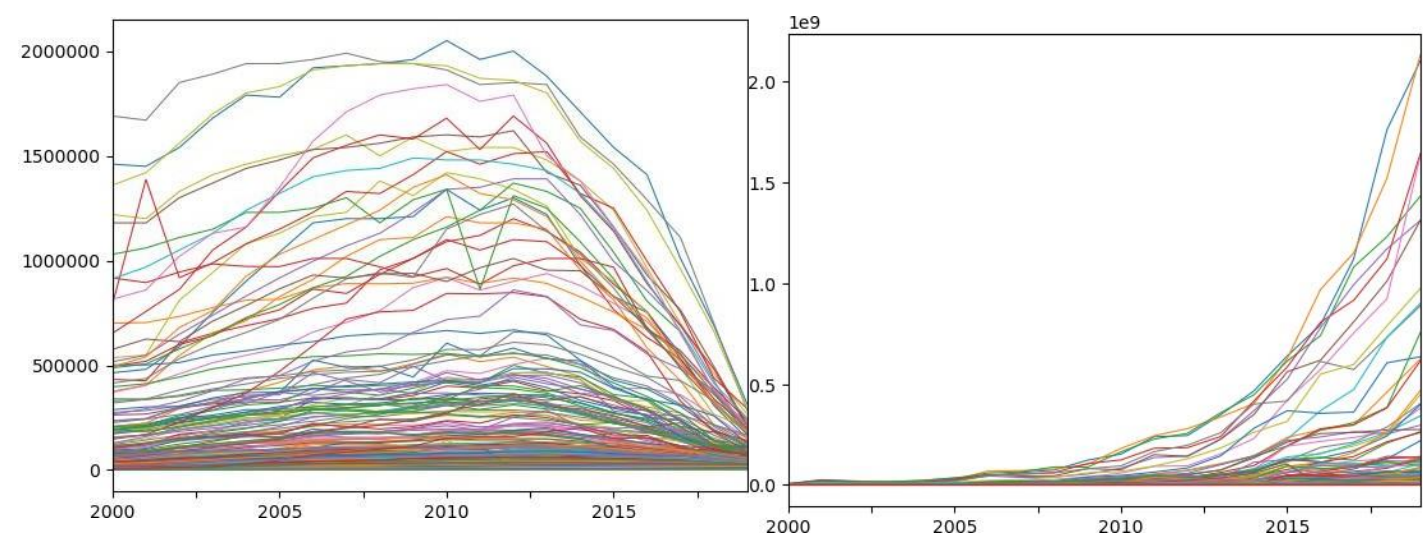

Figure 4(c). For HSI in academia.

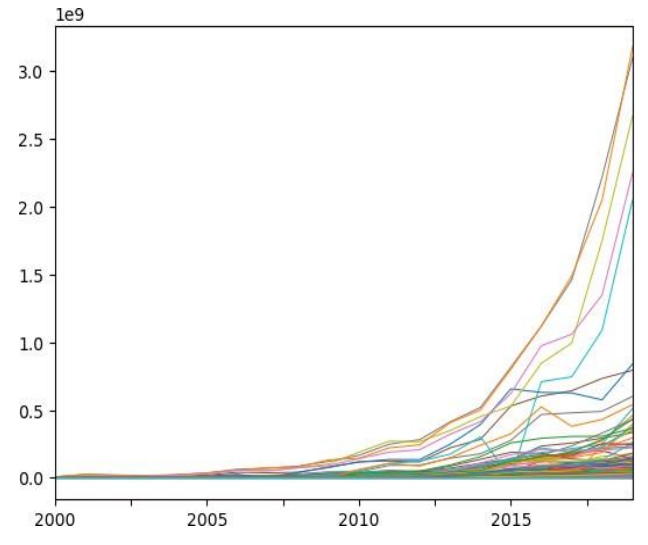

Figure 4(b). For $U A V$ in web.

Figure 4. Trend curves for technology-related entities for two technologies of interest in two different document collections.

\subsection{Identifying Technology-Relevant Entities}

The final process is the identification of technology-relevant entities using the trend curves extracted from the previous section. The candidates are filtered by the combined value of two 
International Journal of Web \& Semantic Technology (IJWesT) Vol.11, No.1/2/3, July 2020

features: total_sum representing the highest trend curves for academia and moving_gradient representing the highest growth rate at a given interval for web. Total_sum is calculated as the normalized sum of the frequencies used in the trend curve. Moving_gradient is calculated as the maximum normalized average gradient, where the average gradient is calculated for each timeslot using the set time window, which is set to five in this experiment; time windows over 2000 2019 are reduced to the limit of the extracted data to deal with over/underflow problems. The frequency values vary greatly from 0 to more than $2.0 \mathrm{e} 9$; therefore $\operatorname{logscale}$ values are used to reduce the differences between them. The algorithm uses the weighted sum of both total_sum and moving_gradient to identify top $\mathrm{n}=100$ entities from both academia and web. Datasets show distinctive differences in their patterns as shown in Figure 4; academia shows plateaued curves while web shows exponentially growing curves. More weight is given to the feature for the dataset it's more relevant to; total_sum $=0.75$ and moving_gradient $=0.25$ for academia and the reverse for web as shown in Figure 5.

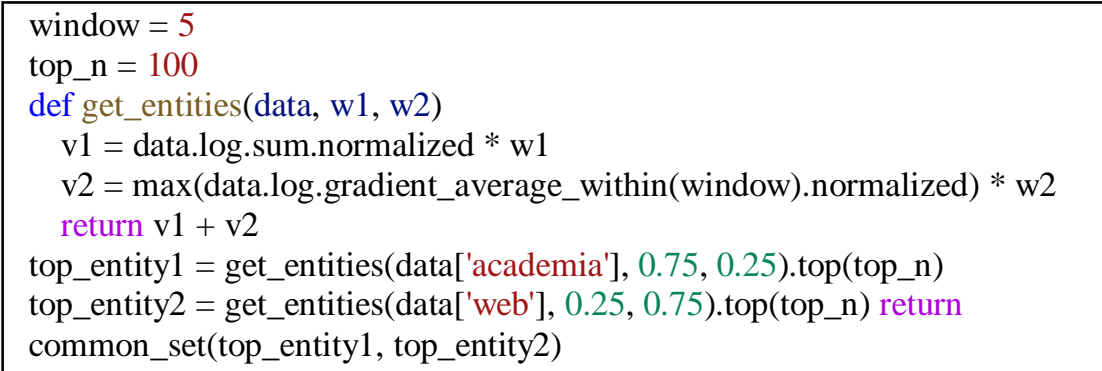

Figure 5. Pseudocode for identifying technology-relevant entities.

Technology-relevant entities are identified from the common denominator between the two resulting sets to allow remediation of disadvantages from individual forecasts at the expense of individual advantages [8]. Incorporating both academia and web, each representing the earliest and the latest predictive time windows, results in a set of relevant entities related to the technology of interest of both the academic interests and public recognitions. Only the entities in the final ranked list from two datasets are selected as the technology-relevant entities, allowing a different number of entities to be found for each technology.

Table 1. List of 40 Technology-Relevant Entities for $U A V$

\begin{tabular}{|l|l|l|}
\hline 3D modeling & Integration platform & Process philosophy \\
\hline Acoustic location & Library (computing) & Radio navigation \\
\hline Actuator & Model aircraft & Ranging \\
\hline $\begin{array}{l}\text { Architecture description } \\
\text { language }\end{array}$ & Model engine & Real time location system \\
\hline CITES & Model ship & Structured Analysis \\
\hline Configuration design & Modular design & Surveillance \\
\hline Conservation law & Open architecture & System design \\
\hline Continuous integration & Open source & System in package \\
\hline Control line & Open source hardware & System of record \\
\hline $\begin{array}{l}\text { Conversation } \\
\text { (disambiguation) }\end{array}$ & Paper plane & System on a chip \\
\hline Core concern & Privacy & Targeted advertising \\
\hline Data mining & Privacy by design & Vocational education \\
\hline Digital identity & Privacy laws of the United & \\
\hline Environment minister & States & \\
\hline
\end{tabular}


40 for UAV and 39 for HIS appeared in both the datasets and were deemed as the technologyrelevant entities as shown in Table 1 and Table 2. The score used in this stage is not used for evaluation, hence the entities are not ranked and listed in alphabetical order. They include a range of entities, from high-level domain entities such as physics and data mining to technologyspecific topics such as ultrasound and privacy, to even clearly unrelated terms such as History of the Internet and Process philosophy. The found entities are manually inspected to discern the false positives to calculate the precision of the proposed method at identifying the relevant technologies.

Table 2. List of 39 Technology-Relevant Entities for HSI

\begin{tabular}{|l|l|l|}
\hline Acoustics & Digital divide & Page table \\
\hline Base address & Digital electronics & Physical symbol system \\
\hline Black box & Digital recording & Physics \\
\hline Candidate key & Digital video & Remote sensing \\
\hline Channel (communications) & Grid computing & Search data structure \\
\hline $\begin{array}{l}\text { Comparison of network } \\
\text { diagram software }\end{array}$ & History of the Internet & Shift register \\
\hline Computer architecture & Information Age & Simulator \\
\hline CPU design & Information system & Software diversity \\
\hline Data (computing) & Internet forum & State machine \\
\hline Data hierarchy & Machine vision & Ultrasound \\
\hline Data mining & Memory address register & $\begin{array}{l}\text { Value } \\
\text { (computer science) }\end{array}$ \\
\hline Data processing & Memory model (programming) & Web service \\
\hline Digital control & Memory protection & Wireless sensor network \\
\hline
\end{tabular}

\subsection{Utilizing Semantic Similarities to the Target Technology}

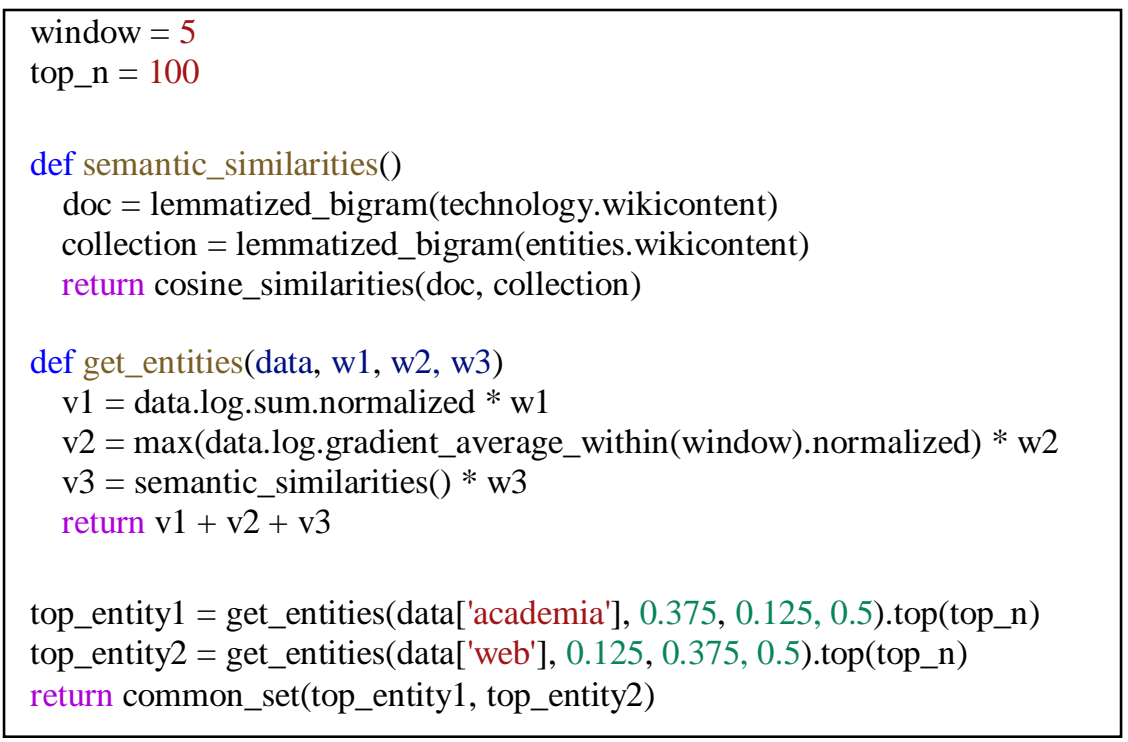

Figure 6. Pseudocode for identifying technology-relevant entities with semantic similarities. 
International Journal of Web \& Semantic Technology (IJWesT) Vol.11, No.1/2/3, July 2020

The entities found in the previous section includes several clearly false results, such as process philosophy, library (computing), history of the internet, information age, and software diversity.

Solely relying on the graph connectivity and trend curve properties do not check for the semantical distances, which can gradually increase as the distances from the source technology increases. The proposed method is augmented so the semantical similarity to the target technology is used in conjunction with the graphical features. Figure 6 shows the overview of the process, where the cosine similarities between the wikicontent section of the technology's and candidate entities' Wikipedia articles are added to the calculation. Gensim3 natural language process function generated a bigram dictionary, and lemmatization is done to extract nouns, verbs, adjectives, and adverbs from it using the spaCy4 English model as done in the author's previous work [19]. Cosine similarities between the technology and candidate entities are then used to represent the semantic similarities between them, which are weighted equally to the sum of total_sum and moving_gradient.

\section{RESUltS AND DISCUSSIONS}

The manual analysis showed that 35 out of the 40 entities found for UAV are relevant, resulting in a precision value of 0.875 . Most of the entities fall under six major categories as shown in Table 3: 1) eight physical components such as actuator, 2) eight vehicle design methods such as 3D modeling, 3) four navigational features such as radio navigation, 4) four surveying functions such as CITES which stands for Convention on International Trade in Endangered Species, 5) six privacy concerns such as digital identity, 6) three other unmanned vehicles, and two uncategorized entities.

Table 3. List of 35 Manually Selected Entities for UAV.

\begin{tabular}{|l|c|c|c|l|}
\hline \multicolumn{1}{|c|}{ Categories } & \multicolumn{4}{|c|}{ Technology-relevant Entities } \\
\hline $\begin{array}{l}\text { Physical } \\
\text { Components }\end{array}$ & Actuator & Control line & Model engine & $\begin{array}{l}\text { Modular } \\
\text { design }\end{array}$ \\
\cline { 2 - 5 } & $\begin{array}{l}\text { Open source } \\
\text { hardware }\end{array}$ & System in package & System of record & $\begin{array}{l}\text { System on a } \\
\text { chip }\end{array}$ \\
\hline $\begin{array}{l}\text { Vehicle Design } \\
\text { Methods }\end{array}$ & 3D modeling & $\begin{array}{l}\text { Architecture description } \\
\text { language }\end{array}$ & $\begin{array}{l}\text { Configuration } \\
\text { design }\end{array}$ & $\begin{array}{l}\text { Continuous } \\
\text { integration }\end{array}$ \\
\cline { 2 - 5 } & Open architecture & Open source & Structured Analysis $\begin{array}{l}\text { System } \\
\text { design }\end{array}$ \\
\hline $\begin{array}{l}\text { Navigational } \\
\text { Features }\end{array}$ & Integration platform & Radio navigation & Ranging & $\begin{array}{l}\text { Real time } \\
\text { location } \\
\text { system }\end{array}$ \\
\hline $\begin{array}{l}\text { Surveying } \\
\text { Functions }\end{array}$ & Acoustic location & CITES & $\begin{array}{l}\text { Environment } \\
\text { minister }\end{array}$ & Surveillance \\
\hline Privacy Concerns & Core concern & Digital Identity & Privacy & $\begin{array}{l}\text { Privacy by } \\
\text { design }\end{array}$ \\
\cline { 2 - 5 } & $\begin{array}{l}\text { Privacy laws of the } \\
\text { United States }\end{array}$ & Privacy Policy & & \\
\hline $\begin{array}{l}\text { Other Unmanned } \\
\text { Vehicles }\end{array}$ & Model aircraft & Model ship & Paper plane & \\
\hline Uncategorized & Data mining & Targeted advertising & & \\
\hline
\end{tabular}

The uncategorized technology-relevant entities show hidden connections. Targeted advertising is a marketing strategy optimizing ads to the specific audiences and is mostly employed in the cyberspace, while UAV provides a physical advertising medium in the air capable of following 
the movement of target audiences over a long period; UAV also allows an easier generation of target-specific contents as well as cheaper aerial accessibility. Data mining is a combination of computer science and statistics seemingly unrelated to UAV, but the increasing number of largescale datasets such as GIS generated by drones leads to an increased need for data mining to process the raw data.

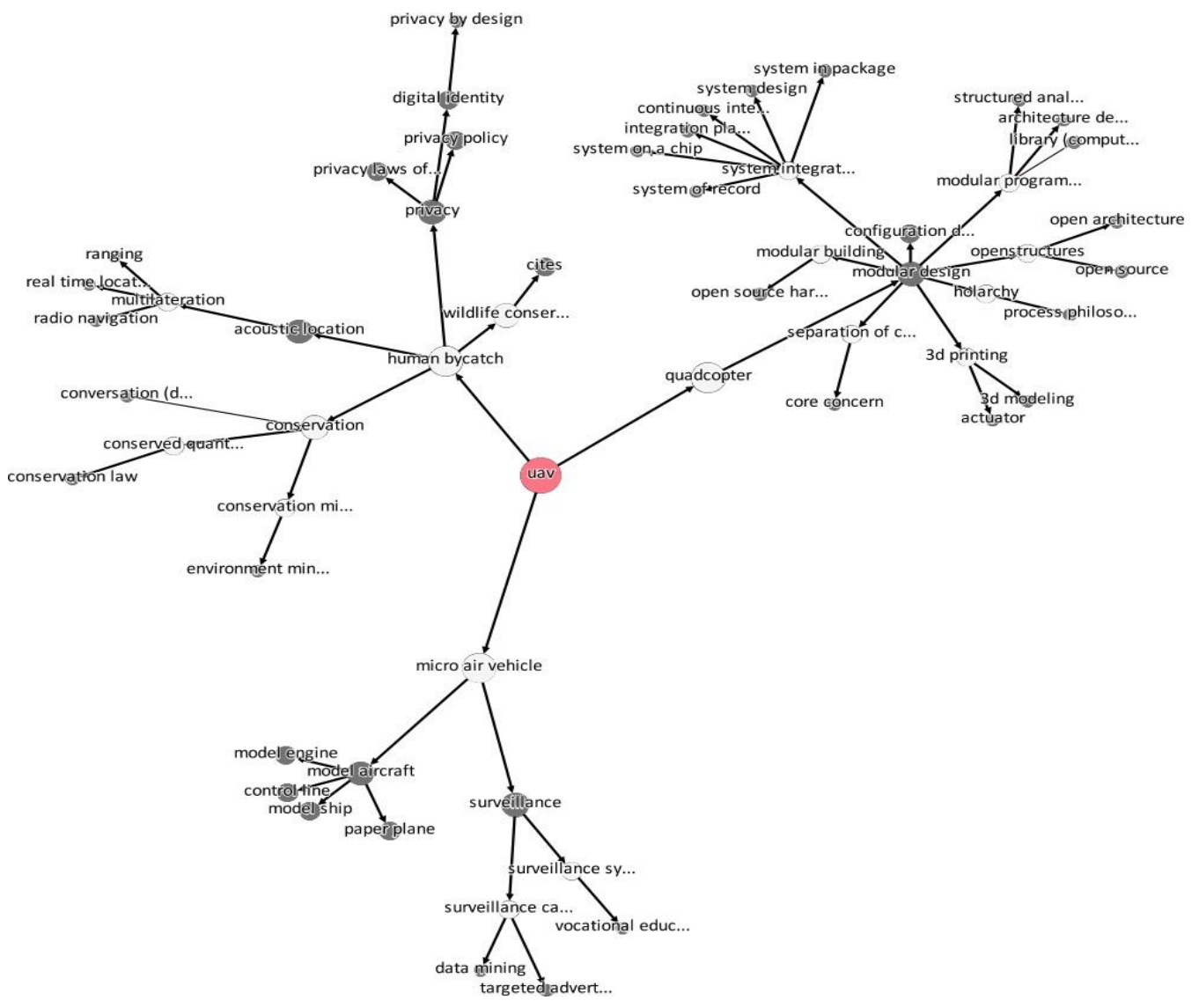

Figure 7. Visualization of paths to the technology-relative entities for $U A V$.

Figure 7 visualizes Wikipedia articles in a directed graph, where entities are linked by their seealso relationships with diminishing node size with longer distances from the seed. The nonrelevant entities acting as a pathway are not colored to distinguish them, while the technology of interest, is colored red to signify the root node in the graph. The tree graph is divided by branches from quadcopter for UAV design and modeling, from human bycatch for navigation and privacy, and micro air vehicle for model and surveillance. The branches do not represent the human categorization; privacy and surveillance branches are far from each other even though the former is the result of the capability of the latter. This suggests that the entities are not necessarily grouped by their graphical structure, nor by their conceptual similarities. CITES, which stands for the Convention on International Trade in Endangered Species of Wild Fauna and Flora, is not related to the surveillance branch, supporting this claim. The graph also explains the existence of seemingly unrelated entities, Conversation (disambiguation) and conservation law; both are connected to the conservation node suggesting that while the former is included as a precaution for mistaking it for conversation, while the latter represents its use in the physics domain.

The manual analysis for HSI resulted in a much lower accuracy of 0.5385 , showing only 21 out of the 39 entities as relevant. The majority of the entities are about the actual process of HSI as 
International Journal of Web \& Semantic Technology (IJWesT) Vol.11, No.1/2/3, July 2020

shown in Table 4, with eleven related to the data acquisition and preprocessing such as digital video and simulators and eight related to the technical and computational methods used during the process such as digital divide and shift register. Two of the remaining entities are acoustics and ultrasound related to the sound.

Table 4. List of 21 Manually Selected Entities for HSI.

\begin{tabular}{|l|c|c|c|c|}
\hline \multicolumn{1}{|c|}{ Categories } & \multicolumn{4}{|c|}{ Technology-relevant Entities } \\
\hline \multirow{2}{*}{$\begin{array}{c}\text { Data Acquisition } \\
\text { / Preprocessing }\end{array}$} & Data (computing) & Data mining & Data processing & Digital electronics \\
\cline { 2 - 5 } & Digital recording & Digital video & Machine vision & Remote Sensing \\
\cline { 2 - 5 } & Simulator & Web service & $\begin{array}{c}\text { Wireless sensor } \\
\text { network }\end{array}$ & \\
\hline $\begin{array}{c}\text { Technical and } \\
\text { Computationl } \\
\text { Components }\end{array}$ & $\begin{array}{c}\text { Channel } \\
\text { (commu } \\
\text { nication } \\
\text { s) }\end{array}$ & $\begin{array}{c}\text { Comparison of } \\
\text { network diagram } \\
\text { software } \\
\text { architecture }\end{array}$ & Digital divide \\
\cline { 2 - 5 } & $\begin{array}{c}\text { Grid computing } \\
\text { Memory address } \\
\text { register }\end{array}$ & Shift register & State machine \\
\hline $\begin{array}{l}\text { Non-invasive } \\
\text { sensing }\end{array}$ & Acoustics & Ultrasound & & \\
\hline
\end{tabular}

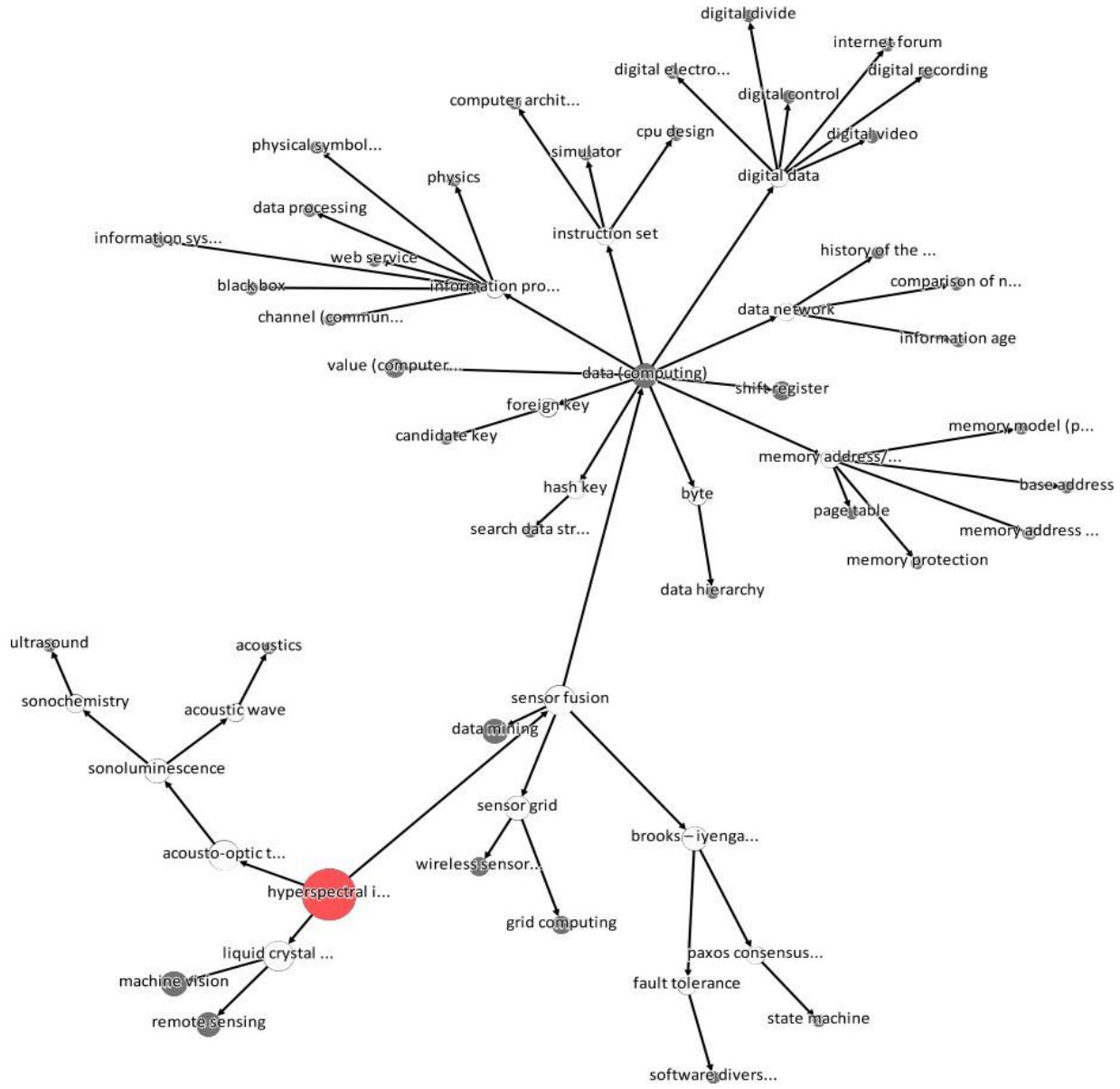

Figure 8. Visualization of paths to the technology-relative entities for HSI. 
The differences in the accuracy can be explained by the skewness of their propagation patterns. Figure 7 for UAV shows a more balanced entity propagation - design perspective, conservation/privacy perspective, and use of micro-size vehicles. On the other hand, the tree graph in Figure 8 for HSI is more skewed towards data (computing) which has a high connection with other entities having less relationship with HSI; 17 out of the 18 unrelated entities were identified from its branch. This shows the danger of utilizing entities with too broad spectrums, where the innate connection to the technology of interest is lost, leading to highly unrelated entities such as history of the internet or physics. Data mining is more closely related to HSI not only in the graph but also in context, as it is a data analysis technique. More layers are used compared to the related multispectral imaging, increasing the necessity of data mining techniques. Two sound-related entities connected to the root node through sono luminescence seem unrelated, but acoustics and ultrasound are connected to HSI as they are the non-invasive remote sensing approaches sharing the same goal of collecting information without making physical contact.

Semantic similarities were utilized to validate the premise that the proposed approach can benefit from combining other forms of connections between technologies and related entities. Applying equal weights to the trend curves and semantic similarities result in the top 100 candidates from each dataset to share more common entities, resulting in more entities found; UAV and HSI respectively showed 62 and 40 relevant entities. The effect of semantic similarities used in conjunction with the trend curves was analyzed for HSI to showcase the possible performance gains. Table 5 shows the technology-relevant entities found for HSI, with newly identified entities in bold. False results such as history of the internet and physics were not removed from the list while others such as process philosophy and library (computing) were successfully removed. This indicates that unrelated entities can be filtered given that the semantic similarity is not overwhelmed by trend curve properties. The result showed an increased accuracy value of 0.65 with manual inspection, where 11 entities were replaced with 12 newly identified entities. More than half of the removed ones, 6 out of 11, were manually deemed relevant, but more technology-relevant entities were newly identified to make up for the loss; 10 out of the 12 were deemed relevant. The majority of the new entities such as imaging spectrometer have connections to the HSI, validating the use of semantic similarities in identifying technology-relevant entities.

Table 5. List of 40 Technology-Relevant Entities for HSI with semantic similarities. Entities found only with semantic similarities are in bold.

\begin{tabular}{|c|c|c|}
\hline Acoustics & Grid computing & Physics \\
\hline Black box & History of the Internet & Preclinical imaging \\
\hline Chemical imaging & Imaging spectrometer & Remote sensing \\
\hline Computer architecture & Information Age & Sensor fusion \\
\hline CPU design & Information processing & Simulator \\
\hline Cyberspace & Information system & Spectral imaging \\
\hline Data (computing) & Internet forum & State machine \\
\hline Data mining & Machine vision & Ultrasonics \\
\hline Data processing & Memory allocation & Ultrasound \\
\hline Digital control & Memory protection & $\begin{array}{l}\text { Value } \\
\text { (computer science) }\end{array}$ \\
\hline Digital divide & Multispectral image & Web service \\
\hline Digital electronics & Optical microscopy & Wireless sensor network \\
\hline Digital video & Page table & \\
\hline Full spectral imaging & Physical symbol system & \\
\hline
\end{tabular}


Table 6. List of 12 Newly Identified Technology-Relevant Entities for HSI with their categories.

\begin{tabular}{|c|c|c|l|}
\hline Categories & Technology-relevant Entities & \\
\hline Data & Information Processing & & \\
\hline Components & Imaging spectrometer & Sensor fusion & \\
\hline Non-invasive sensing & Optical microscopy & Ultrasonics & \\
\hline Spectral imaging & Full spectral imaging & Multispectral image & Spectral imaging \\
\hline Applications & Chemical imaging & Preclinical imaging & \\
\hline Unrelated & Cyberspace & Memory allocation & \\
\hline
\end{tabular}

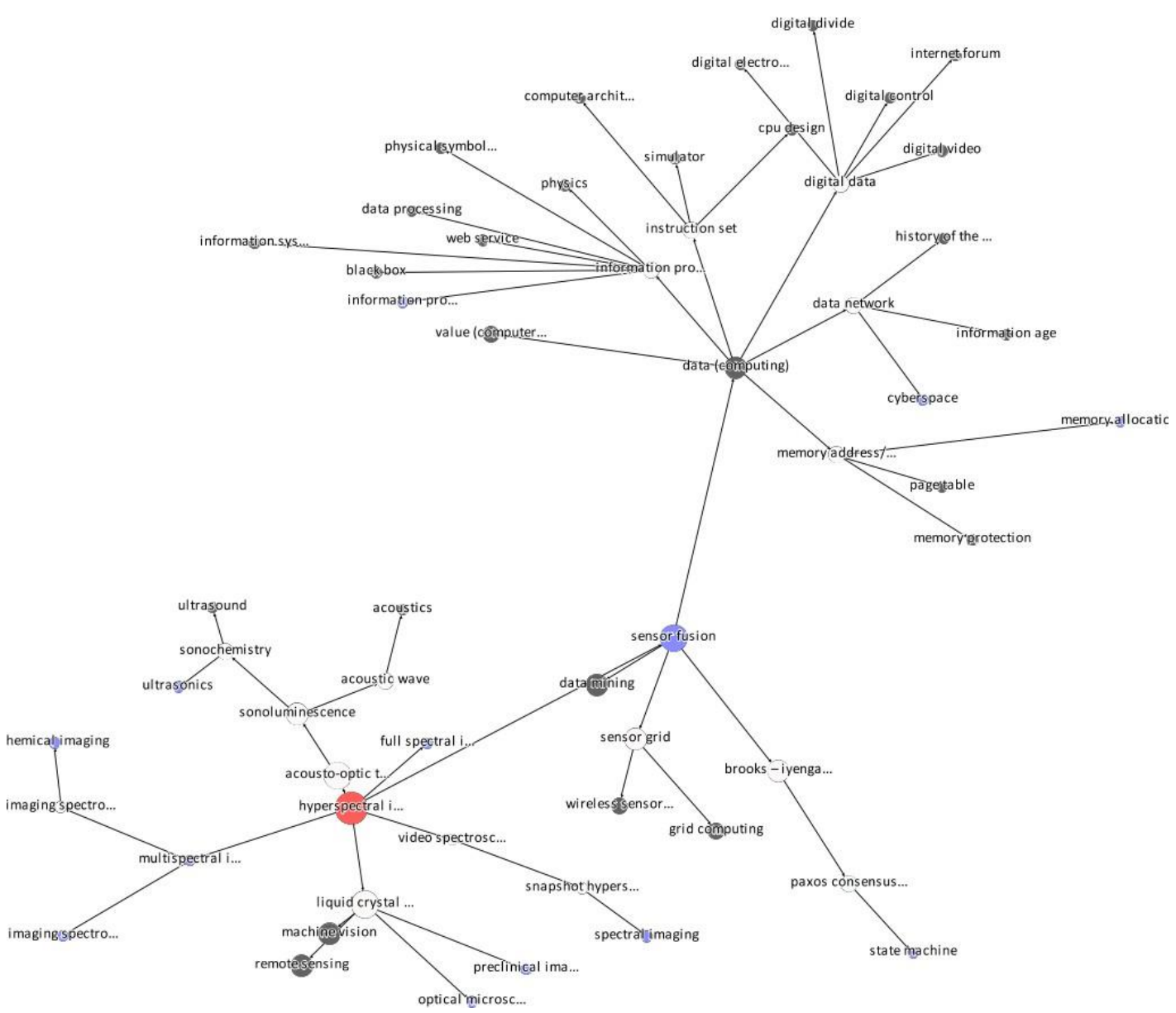

Figure 9. Visualization of paths to the technology-relative entities for HIS with semantic similarities, where newly introduced entities are shown in blue.

Table 6 shows the categories of the newly identified entities introducing introduced two categories with a more direct connection to $\mathrm{HSI}$; spectral imaging techniques such as multispectral image and applications of the hyperspectral imaging such as preclinical imaging. There were also several interesting findings as well; optical microscopy is one of the early forms of non-invasive sensing technique which can also be used to generate imaging data on which HSI operates. Sensor fusion was only used as a pathway in Figure 8 even though it is a necessary technique to generate contiguous spectral band data on 
International Journal of Web \& Semantic Technology (IJWesT) Vol.11, No.1/2/3, July 2020

which hyperspectral imaging is run, which was successfully captured with the introduction of semantic similarities as shown in Figure 9. The path visualization indicates the graph is now more balanced, having move

branches in the seed node at the expense of branches from data (computing) node. None of the entities directly connected to the root node in Figure 7 and Figure 8 were identified as technology-relevant entities. Not necessarily by design, this validates the ability of the method to identify remotely-connected entities while being able to capture closely-connected entities as well as shown in Figure 9 where $40 \%$ of the direct descendants of the root node were identified as technology-relevant entities.

\section{CONCLUSION}

The authors propose a method of identifying any type of entities related to a given technology based on their trend curves. The results showed that the entities with recursive relationships in Wikipedia have connections to the target technology not directly observed by either of their encyclopedic descriptions. Case studies revealed that the proposed method can identify entities related to the given technology with hidden relationships. This discovery suggests that the tacit relationships between semantically and syntactically distant technologies can be captured automatically from existing datasets. This opens a path of technology forecasting utilizing the growth of other relevant technologies.

One of the issues for the proposed method is the computational delay when generating the trend curves. The computational complexity is low for trend extraction with $\mathrm{O}(\mathrm{nt})$ where $\mathrm{n}$ is the number of trends and $t$ is the number of years analyzed. The computation time suffers mostly from the Google search engine API restrictions; the number of query requests is limited to one per 100 seconds. With 20 years to analyze in two different datasets, trend curves for technologyrelevant entities can be extracted in over 55.5 hours on a standard computer. Another issue that has an influence on the result is that the relatedness between technology and entities is defined as a binary, treating all related entities equally. The structural similarities between them were omitted in the proposed method, rendering it hard to distinguish how related an entity is to the target technology, thus resulting in poor precision for HSI due to the entities related to data (computing) polluting the entity pool. Future work includes the combination of trend curves with graphical similarities. Incorporating graphical similarities would allow the method to selectively filter for a specific degree of similarities. Utilizing the extracted entities would allow technology forecasting based on the development stages and needs of the related technologies reflected by their trend curves. The future works would also include experimenting on a known case of technology impacted by seemingly unrelated entities to evaluate whether the proposed method can detect such entities beforehand, and proposing different weighting schemes to enhance the accuracy of the approach.

\section{ACKNOWLEDGMENTS}

This work was supported by the Deep Neural Networks for Forensic and Industry Classifications funded by the Industry Advisory Board (IAB) of an NSF Industry-University Cooperative Research Center (IUCRC) under Grant No. DFII-1903-USA.

\section{REFERENCES}

[1] A. Segev, S. Jung, and S. Choi, "Analysis of Technology Trends Based on Diverse Data Sources," IEEE Trans. Serv. Comput., vol. 2015 Vol.8, no. 06, pp. 903-915, Dec. 2015, doi: 10.1109/TSC.2014.2338855. 
International Journal of Web \& Semantic Technology (IJWesT) Vol.11, No.1/2/3, July 2020

[2] P. Durance and M. Godet, "Scenario building: Uses and abuses," Technol. Forecast. Soc. Change, vol. 77, no. 9, pp. 1488-1492, Nov. 2010, doi: 10.1016/j.techfore.2010.06.007.

[3] K. C. Green and J. S. Armstrong, "Structured analogies for forecasting," Int. J. Forecast., vol. 23, no. 3, pp. 365-376, Jul. 2007, doi: 10.1016/j.ijforecast.2007.05.005.

[4] N. Dalkey and O. Helmer, "An Experimental Application of the DELPHI Method to the Use of Experts,” Manag. Sci., vol. 9, no. 3, pp. 458-467, Apr. 1963, doi: 10.1287/mnsc.9.3.458.

[5] S. Li, E. Garces, and T. Daim, "Technology forecasting by analogy-based on social network analysis: The case of autonomous vehicles," Technol. Forecast. Soc. Change, vol. 148, p. 119731, Nov. 2019, doi: 10.1016/j.techfore.2019.119731.

[6] F. Zwicky, "The Morphological Approach to Discovery, Invention, Research and Construction," in New Methods of Thought and Procedure, Berlin, Heidelberg, 1967, pp. 273-297, doi: 10.1007/978-3642-87617-2_14.

[7] J. P. Martino, Technological forecasting for decision making, 3rd ed. New York: McGraw-Hill, 1993.

[8] J. S. Armstrong, "Forecasting by Extrapolation: Conclusions from 25 Years of Research," Inf. J. Appl. Anal., vol. 14, no. 6, pp. 52-66, Dec. 1984, doi: 10.1287/inte.14.6.52.

[9] D. Henton and K. Held, "The dynamics of Silicon Valley: Creative destruction and the evolution of the innovation habitat," Soc. Sci. Inf., vol. 52, no. 4, pp. 539-557, Dec. 2013, doi: $10.1177 / 0539018413497542$.

[10] O. Dedehayir and M. Steinert, "The hype cycle model: A review and future directions," Technol. Forecast. Soc. Change, vol. 108, pp. 28-41, Jul. 2016, doi: 10.1016/j.techfore.2016.04.005.

[11] J. S. Armstrong and F. Collopy, "Error measures for generalizing about forecasting methods: Empirical comparisons," Int. J. Forecast., vol. 8, no. 1, pp. 69-80, Jun. 1992, doi: 10.1016/01692070(92)90008-W.

[12] D. M. Blei and J. D. Lafferty, "Dynamic Topic Models," in Proceedings of the 23rd International Conference on Machine Learning, New York, NY, USA, 2006, pp. 113-120, doi: 10.1145/1143844.1143859.

[13] N. Takeda, Y. Seki, M. Morishita, and Y. Inagaki, "Evolution of Information Needs based on Life Event Experiences with Topic Transition," in Proceedings of the 40th International ACM SIGIR Conference on Research and Development in Information Retrieval, Shinjuku, Tokyo, Japan, Aug. 2017, pp. 1009-1012, doi: 10.1145/3077136.3080703.

[14] Y. Cho and T. Daim, "OLED TV technology forecasting using technology mining and the Fisher-Pry diffusion model," Foresight, vol. 18, no. 2, pp. 117-137, Jan. 2016, doi: 10.1108/FS- 08-2015-0043.

[15] A. Segev, "Circular context-based semantic matching to identify web service composition," in Proceedings of the 2008 international workshop on Context enabled source and service selection, integration and adaptation organized with the 17th International World Wide Web Conference (WWW 2008) - CSSSIA '08, Beijing, China, 2008, pp. 1-5, doi: 10.1145/1361482.1361489.

[16] M. Gusenbauer, "Google Scholar to overshadow them all? Comparing the sizes of 12 academic search engines and bibliographic databases," Scientometrics, vol. 118, no. 1, pp. 177-214, Jan. 2019, doi: 10.1007/s11192-018-2958-5.

[17] M. de Kunder, "The size of the World Wide Web (The Internet)," Mar. 07, 2020. https://www.worldwidewebsize.com/.

[18] A. Segev, C. Jung, and S. Jung, "Analysis of Technology Trends Based on Big Data," in 2013 IEEE International Congress on Big Data (BigData Congress), Jun. 2013, pp. 419-420, doi: 10.1109/BigData.Congress.2013.65.

[19] S. Jung and W. C. Yoon, "An alternative topic model based on Common Interest Authors for topic evolution analysis," J. Informetr., vol. 14, no. 3, p. 101040, Aug. 2020, doi: 10.1016/j.joi.2020.101040. 


\section{AUTHORS}

Sukhwan Jung is a Postdoc in the Department of Computer Science, University of South Alabama. He earned his Ph.D. in Knowledge Service Engineering at KAIST in 2020.

Rachana Reddy Kandadi is a Master's degree student in the Department of Computer Science, University of South Alabama. She earned her undergraduate degree from JNTUH in Computer Science and Engineering.

Rituparna Datta is a Computer Research Associate-II in the Department of Computer Science, University of South Alabama. Prior to that, he was an Operations Research Scientist in Boeing Research \& Technology (BR\&T), BOEING, Bangalore.

Ryan Benton is an Assistant Professor in the Department of Computer Science at the University of South Alabama. His research interests lies in the fields of data mining and machine learning, with a current focus upon advanced pattern mining methods, novel graph mining algorithms, and applied applications.

Segev Aviv is an Associate Professor in the Department of Computer Science at the University of South Alabama. His research interest is looking for the DNA of knowledge, an underlying structure common to all knowledge, through analysis of knowledge models in natural sciences, knowledge processing in natural and artificial neural networks, and knowledge mapping between different knowledge domains.
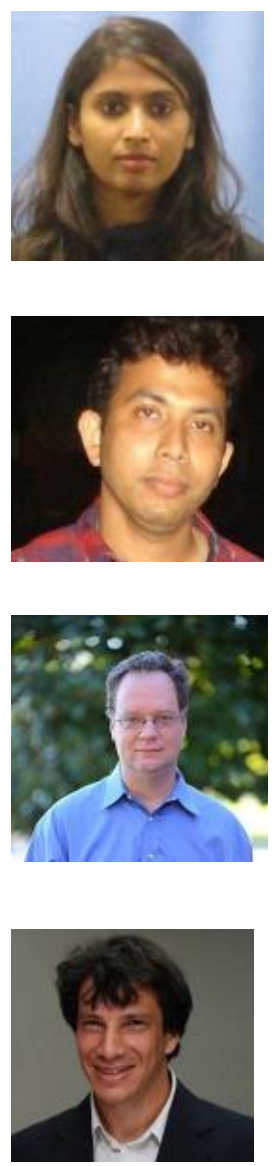